\title{
Are patients with dementia treated as well as patients without dementia for hypertension, diabetes, and hyperlipidaemia?
}

Johannes Müther, Heinz-Harald Abholz, Birgitt Wiese, Angela Fuchs, Anja Wollny and Michael Pentzek

\author{
ABSTRACT \\ Background \\ Patients suffering from dementia are at risk of being \\ treated differently by GPs from patients without it. \\ Explanations for this could be stigmatisation, treatment \\ with a palliative approach, and the result of the disease \\ process. \\ Aim \\ To ascertain whether patients with dementia are \\ treated differently, the index diseases of hypertension, \\ diabetes, and hyperlipidaemia were used to measure \\ care. \\ Design of study \\ Retrospective matched control study.

\section{Setting} \\ German general practice. \\ Method \\ Sixteen GP practices recruited all their patients with \\ dementia and at least one of the index diseases. \\ Patients without dementia but only the index diseases \\ were matched for age, sex, index disease, and \\ practice, resulting in 216 pairs of patients with and \\ without dementia. From the files, blood pressure, blood \\ sugar/glycated haemoglobin, cholesterol, the dates of \\ measurement, the number of doctor-patient contacts, \\ and the prescribed medication to treat the three \\ conditions under scrutiny were documented. For \\ analysis, $t$-tests and $\chi^{2}$-tests were used.

\section{Results} \\ No differences were found in treatment outcomes \\ between the two patients groups, except one \\ significant difference: one of the two documented \\ systolic blood pressure values is lower in the dementia \\ group. Furthermore, patients with dementia more often \\ do not receive any medication or are treated with low- \\ priced medications for hypertension (nearly significant).

\section{Conclusion} \\ GPs do not seem to treat patients with dementia \\ differently. The use of lower-priced antihypertensive \\ medication could be the only indication for some kind \\ of difference in approach. \\ Keywords \\ attitude of health personnel; comorbidity; dementia; \\ family practice; general practitioners; quality; \\ stigmatisation; treatment.
}

\section{INTRODUCTION}

There has always been speculation that patients with dementia, a socially stigmatising disease, receive inferior treatment for their general health compared to patients without dementia. However, the few studies that have confronted this topic, using very different methodical and empirical approaches, have produced fairly inconsistent results. However, a recent systematic review on the quality of health care of physical illnesses in mentally ill patients, compared to those without mental illness, showed that most of the studies found inequity. ${ }^{1}$ These studies emanated predominantly from the US, as well from the UK, Finland, and the Netherlands ${ }^{2-9}$ and, for the most part, they were secondary evaluations of routine data. There is also a scarcity of studies surrounding other socially stigmatising diseases such as schizophrenia or depression. One of these studies uses an approach that is very similar to the one chosen in the current study..$^{10}$ It was a matchedcontrol study that focused on the preventive treatment of cardiovascular risk factors in

JMüther, GP, Elsdorf/Cologne, Germany. H-H Abholz, MD, professor, head of department; A Fuchs, senior researcher; M Pentzek, PhD, senior researcher, University Hospital Düsseldorf, Department of General Practice, Heinrich-HeineUniversity Düsseldorf, Germany. A Wollny, senior researcher, Institute for Primary Medical Care, University of Rostock, Medical Faculty, Rostock, Germany. B Wiese, Institute for Biometry, Hannover Medical School, Hannover, Germany.

Address for correspondence

Professor Heinz-Harald Abholz, Abteilung für Allgemeinmedizin, Universitätsklinikum Düsseldorf, Moorenstr. 5, 40225 Düsseldorf, Germany. E-mail: abholz@med.uni-duesseldorf.de

Submitted: 15 February 2010; Editor's response: 30 March 2010; final acceptance: 12 April 2010

(c)British Journal of General Practice 2010; 60: 671-674.

DOI: 10.3399/bjgp10X515395 


\section{How this fits in}

There are only a few studies on patients with 'stigmatised diseases' such as dementia, schizophrenia, drug abuse, and depression, and the care of these patients concerning comorbidities or preventive measures. The results of these studies are conflicting. In this matched control study it was shown that German GPs do not seem to follow social stigmatisation when caring for hypertension, diabetes, and lipid disorders in patients with dementia.

individuals with a diagnosis of schizophrenia compared to the care of patients without this diagnosis. In this study, ${ }^{10}$ based in general practices in England, 'negligence' was found in the treatment of cardiovascular risk factors in patients with a diagnosis of schizophrenia.

\section{Research question}

This study aimed to find out whether patients who have been identified by their family doctor as having dementia are treated differently from patients who are classified as 'non-demented'. Treatment parameters were chosen for hypertension, diabetes, and hyperlipidaemia, in order to gather measurable data on the quality of treatment.

\section{METHOD}

Sixteen of the 25 invited teaching practices of the Department of General Practice, Universitaetsklinikum Duesseldorf, were recruited into the study. GPs had to identify their patients from their electronic data files, taking the following steps:

1. A list was compiled of all patients who had visited

Table 1. Characteristics of the sample.

\begin{tabular}{lcc} 
Variable & Dementia $(n=216)$ & No dementia $(n=216)$ \\
\hline Mean age, years (SD) & $82.7(6.2)$ & $82.2(6.3)$ \\
\hline Females, \% & 77.3 & 77.3 \\
\hline Mean number of comorbidities (SD) & $2.3(1.2)$ & \\
\hline Dependency, \% & & \\
Completely independent & 5.3 \\
Nearly independent & 22.3 \\
Mildly dependent & 36.9 \\
Seriously dependent & 34.0 \\
Completely dependent & 1.5 \\
\hline Cognition, \% ${ }^{\mathrm{b}}$ & \\
No impairment & 0.5 \\
Mild impairment & 15.4 \\
Mild dementia & 31.7 \\
Moderate dementia & 30.2 \\
Severe dementia & 22.3 \\
\hline Anti-dementia drug prescribed, \% ${ }^{\mathrm{c}}$ & \\
Yes & 37.0 \\
No & 63.0 \\
\hline
\end{tabular}

${ }^{a} 10$ missing data. ${ }^{b} 14$ missing data. ${ }^{\circ} 8$ missing data. $S D=$ standard deviation. the practice in the previous 3 months who were aged $\geq 65$ years.

2. From that list, all patients who had been registered at the practice for less than 2 years, or who had a terminal disease that meant death in the following 12 months was a possibility were excluded. Patients without any of the three studied comorbidities were also excluded from the list (= list of patients after application of criteria for exclusion and inclusion).

3. Patients who had been diagnosed with dementia by the doctor were included in the list. Doctors had to note down the comorbidities of every patient (= list of patients with dementia with any of the three comorbidities).

4. A list of matched pairs was compiled using the list of patients from step 1. Within each practice, patients without dementia were matched with those with dementia on the basis of sex, age ( \pm 3 years), and comorbidity diagnoses.

On a prepared form, the GPs documented the following information covering the previous 2 years for the patients with dementia: dementia status (subjective rating of the GP on a scale: no impairment, mild impairment, mild dementia, moderate dementia, severe dementia), degree of dependency in daily life (subjective rating, see Table 1 for categories), living conditions (alone, with partner or other relatives, in a nursing home). For every patient, GPs had to document age, sex, number of doctor-patient contacts during the last 3 months, blood pressure, blood sugar, and blood cholesterol, as well as the relevant dates of measurement and prescribed medication (classified into categories: $0=$ no medication; 1 = standard medication; 2 and $3=$ more expensive medications).

To analyse the differences between the two groups, $t$-tests and $\chi^{2}$ tests (two-tailed) were performed. Only those pairs with complete data were analysed, so the sample size differs according to which parameter is analysed. The overall significance level was set to $P<0.01$, in order to partially account for the multiple testing.

\section{RESULTS}

The 16 teaching practices included a total of 216 patient pairs (3-28 per practice). Patient characteristics are shown in Table 1.

\section{Outcome parameters}

There was one significant difference between patients with and without dementia in terms of the parameters relating to blood pressure, blood cholesterol, blood sugar, and glycated haemoglobin 
$\left(\mathrm{HbA}_{1 \mathrm{c}}\right)$ : the second documented systolic blood pressure value is significantly lower within the group of patients with dementia (Table 2).

With regard to the intervals between the control dates of the treatment indicators - blood pressure, cholesterol, blood sugar, and $\mathrm{HbA}_{1 \mathrm{c}}$ - there were no significant differences. The same applies to the number of doctor-patient contacts (data not included here).

\section{Medication}

One result related to medication approached the significance level $(P=0.013$ ) (Table 3). Patients with dementia were less likely to receive antihypertensive drugs at all, or were more often treated with group 1 substances (standard and low-priced medication: diuretic, beta-blocker, angiotensin-converting enzyme inhibitors).

\section{Missing values}

The frequency of missing data across all parameters in both patient groups was analysed on the basis of each respective comorbidity. While parameters for diabetes (blood sugar and $\mathrm{HbA}_{1 \mathrm{c}}$ ) and hyperlipidaemia (cholesterol) showed no differences with regard to missing data, the blood pressure parameters were more frequently documented in the non-demented patient group (data not included). Further exploratory analyses revealed that patients with dementia with missing hypertension parameters tended to be in the more advanced stages of dementia, while patients with dementia with documented blood pressure values were more often classified as having 'mild dementia'.

\section{DISCUSSION}

\section{Summary of main findings}

No significant differences were found in the treatment of the selected comorbidities or their outcomes in the study sample of doctors and patients. The fact that patients with dementia tended to receive no medication, or received more standard, low-priced substances for hypertension does not indicate a lack of quality care because it was not possible to ascertain any disadvantage in terms of blood pressure outcomes. Indeed the low-priced medications tended to be more evidence based than the medications in groups 2 or 3 of the antihypertensives.

The fact that there was a relatively large group of patients who did not undergo antihypertensive therapy can be explained by weight loss, which is common among patients with dementia. This cannot be proved definitively because information regarding the weight of patients was not included in the documentation form.

\section{Table 2. Comparisons for outcome parameters.}

\begin{tabular}{lccc} 
Parameter, mean (SD) & Dementia & No dementia & $P$-value \\
\hline Blood sugar 1 $(n=132)$ & $139.5(54.8)$ & $144.2(69.1)$ & 0.665 \\
\hline Blood sugar 2 $(n=116)$ & $142.3(49.8)$ & $146.4(49.6)$ & 0.661 \\
\hline HbA $_{1 c} 1(n=80)$ & $7.2(1.6)$ & $7.2(1.1)$ & 0.975 \\
\hline HbA 2 2 $(n=58)$ & $7.4(1.4)$ & $7.2(1.3)$ & 0.578 \\
\hline Blood pressure systolic 1 $(n=302)$ & $139.5(21.2)$ & $145.7(20.5)$ & 0.011 \\
\hline Blood pressure diastolic 1 $(n=302)$ & $78.7(11.8)$ & $80.7(9.8)$ & 0.107 \\
\hline Blood pressure systolic 2 $(n=290)$ & $142.9(22.0)$ & $151.7(20.0)$ & $<0.001$ \\
\hline Blood pressure diastolic 2 $(n=290)$ & $81.1(12.9)$ & $82.4(10.4)$ & 0.342 \\
\hline Blood cholesterol 1 $(n=204)$ & $242.5(52.2)$ & $234.6(46.7)$ & 0.252 \\
\hline Blood cholesterol 2 $(n=172)$ & $242.5(46.1)$ & $228.3(45.5)$ & 0.044 \\
\hline
\end{tabular}

$S D=$ standard deviation. ${ }^{\text {at-test }}$.

\section{Strengths and limitations of the study}

On the basis of the study results, it is not possible to establish whether or not patients were treated differently for other comorbidities, especially symptomatic ones. The mentioned comorbidities were chosen for the study because they are common in older people, and metric outcomes of treatment can therefore be measured. The existence of generally accepted treatment standards and objectives also allows judgement of the quality of care, while in other common symptomatic diseases, such as chronic obstructive pulmonary disease or rheumatic diseases, the outcome is more dependent on the nature and progression of the disease itself.

Because it was not possible to carry out a power calculation prior to the study, the possibility that less-significant differences remain undetected cannot be excluded. However, it is important to remember that any differences that were found significant or not - pave the way for better control within the dementia group.

Table 3. Comparisons for medication.

\begin{tabular}{lccc} 
Medication, \% within group & Dementia & No dementia & $P$-value \\
\hline Diabetes $(n=138)$ & & & 0.677 \\
Group 0 drugs & 33.3 & 40.6 & \\
Group 1 drugs & 23.2 & 20.3 & \\
Group 2 drugs & 43.5 & 39.1 & 0.013 \\
\hline Hypertension $(n=362)$ & & & \\
Group 0 drugs & 14.9 & 9.9 & \\
Group 1 drugs & 58.6 & 48.6 & \\
Group 2 drugs & 21.0 & 28.7 & \\
Group 3 drugs & 5.5 & 12.7 & \\
\hline Hyperlipidaemia $(n=216)$ & & & \\
Group 0 drugs & 70.4 & 72.2 & \\
Group 1 drugs & 13.9 & 9.3 & \\
Group 2 drugs & 15.7 & 18.5 & \\
\hline
\end{tabular}

${ }^{a} \chi^{2}$ test. 
Nevertheless, more missing data in terms of blood pressure values in the dementia group point to the possibility of bias. It is possible that the aforementioned finding (lower blood pressure in patients with dementia) could stem from a documentation bias. That is, GPs may tend to document blood pressure in patients with dementia whose blood pressure was more controlled and omit it in patient with less controlled blood pressure. The exploratory analysis of the study supports this hypothesis and may also indicate a less accurate documentation of blood pressure values in advanced stages of dementia. This could be interpreted as an indication of poorer diligence in the care of seriously demented patients.

The participating doctors worked in teaching practices. Their care may be of higher quality than that of typical doctors, and their attitudes towards dementia may be less negative (less stigmatisation). Altogether, this could have introduced a bias.

It may also be significant that the participating doctors only included a relatively small number of patients with dementia into the study and that therefore a process of selection may have occurred. From other studies it is known that recognition and more detailed documentation of dementia in general practice is more likely in advanced stages of dementia. ${ }^{11,12}$ Therefore among the patients not included in the study there may be a number having mild cognitive impairment or mild dementia. These cases would have been identified when applying standardised diagnostic procedures like neuropsychological instruments. However, not identifying these patients is not a real limitation when trying to answer the study question: to find out if GPs treat patients 'seen as demented' differently from matched patients without dementia. This requires the sole inclusion of patients who are definitively seen as demented by their GPs.

Moreover, when the low number of patients with dementia per practice is considered, it is important to remember that only patients aged $\geq 65$ years had to be included, and only those who additionally suffered from one or more of the three comorbidities. This noticeably reduces the group of eligible patients.

\section{Implications for clinical practice}

On this background, and bearing the limitations in mind, the study results can be interpreted as follows: either the study sample doctors were not influenced by any stigma surrounding dementia and treated all of their patients in a thoroughly professional manner. The second, also plausible, hypothesis is that GPs tended to concentrate on the treatment of somatic comorbidities, because treating cognitive decline in dementia is a more difficult task. Support for this hypothesis comes from qualitative studies, ${ }^{13,14}$ according to which GPs may feel more confident treating disorders that they are able to control pharmacologically.

\section{Funding body}

This study was conducted within the German Study on Ageing, Cognition and Dementia in Primary Care Patients (AgeCoDe). AgeCoDe is part of the German Competence Network Dementia (KND) and the German Competence Network Degenerative Dementias (KNDD), and was funded by the German Federal Ministry of Education and Research (grants KND: 01GI0423, 01GI0434; grants KNDD: 01GI0713, 01GI0716).

\section{Ethical approval}

The ethics committee of the Düsseldorf University Hospital has approved this study.

\section{Conflicts of interest}

The authors have stated that there are none.

\section{Discuss this article}

Contribute and read comments about this article on the Discussion Forum: http://www.rcgp.org.uk/bjgp-discuss

\section{REFERENCES}

1. Mitchell AJ, Malone D, Doebbeling CC. Quality of medical care for people with and without comorbid mental illness and substance misuse: systematic review of comparative studies. Br J Psychiatry 2009; 194(6): 491-499.

2. Frayne S, Jewell HH, Miller DR, et al. Disparities in diabetes care. Arch Intern Med 2005; 165(22): 2631-2638.

3. Löppönen M, Räihä I, Vahlberg T, et al. Dementia associates with undermedication of cardiovascular diseases in the elderly. Dement Geriatr Cogn Disord 2006; 22(2): 132-141.

4. Morrison RS, Siu LA. A comparison of pain and its treatment in advanced dementia and cognitively intact patients with hip fracture. $J$ Pain Symptom Manage 2000; 19(4): 240-248.

5. Rodriguez EG, Dodge HH, Birzescu MA, et al. Use of lipid-lowering drugs in older adults with and without dementia: a community-based study. J Am Geriatr Soc 2002; 50(11): 1852-1856.

6. Simpson CR, Hannaford PC, McGovern M, et al. Are different groups of patients with stroke more likely to be excluded from the new UK general medical services contract? A cross-sectional retrospective analysis of a large primary care population. BMC Fam Pract 2007; 8: 56

7. Sloan F, Trogdon JD, Curtis LH, Schulman KA. The effect of dementia on outcomes and process of care for medicare beneficaries admitted with acute myocardial infarction. J Am Geriatr Soc 2004; 52(2): $173-181$.

8. Van der Steen JT, Ooms ME, Adér HJ, et al. Withholding antibiotic treatment in pneumonia patients with dementia. Arch Intern Med 2002; 162(15): 1753-1760.

9. Wagner G, Icks A, Abholz HH, et al. Kosten der Pharmakotherapie internistischer Komorbiditäten bei Patienten mit Demenz in der Hausarztpraxis [Costs of pharmacotherapy of somatic co-morbidities in dementia patients in family practices]. Z Allg Med 2007; 83: $411-416$.

10. Roberts L, Roalfe A, Wilson S, Lester H. Physical health care of patients with schizophrenia in primary care: a comparative study. Fam Pract 2007; 24(1): 34-40.

11. Pentzek M, Wollny A, Wiese B, et al, for the AgeCoDe Study Group. Apart from nihilism and stigma: what influences general practitioners' accuracy in identifying incident dementia? Am J Geriatr Psychiatry 2009; 17(11): 965-975.

12. Valcour VG, Masaki KH, Curb JD, Blanchette PL. The detection of dementia in the primary care setting. Arch Intern Med 2000; 160(19): 2964-2968.

13. Boise L, Camicioli R, Morgan DL, et al. Diagnosing dementia: perspectives of primary care physicians. Gerontologist 1999; 39(4): $457-464$.

14. Hansen EC, Hughes C, Routley G, Robinson AL. General practitioners' experiences and understandings of diagnosing dementia: Factors impacting on early diagnosis. Soc Sci Med 2008; 67(11): 1776-1783. 